\title{
Uso de mallas de polipropileno en el reparo de la hernia inguinal con herida sucia o contaminada
}

\author{
Use of polypropylene meshes in the repair of inguinal hernia with dirty or \\ contaminated wound
}

\author{
Andrés Mendoza-Zuchini ${ }^{1,2}$, Mauricio Pedraza-Ciro ${ }^{1,2}$, Sebastián Sánchez-Ussa', \\ Luis Felipe Cabrera², Jean Pulido², Daniel Gómez ${ }^{3}$ \\ Departamento de Cirugía General, Centro Policlínico Olaya, Bogotá, D.C., Colombia
Departamento de Cirugía General, Universidad El Bosque, Bogotá, D.C., Colombia
Minimally Invasive Surgery Independent Research Group, MISIRG, Bogotá D.C., Colombia.
}

\section{Resumen}

Introducción. La hernioplastia con malla de polipropileno es la técnica de elección para el reparo de las hernias inguinales. Actualmente, existe controversia sobre esta técnica en pacientes con heridas sucias o contaminadas; sin embargo, la evidencia en la literatura médica ha demostrado que su uso puede ser seguro. Los autores presentan su experiencia con las mallas de polipropileno en la cirugía contaminada para cierre de hernias inguinales.

Método. Se realizó un estudio observacional retrospectivo en el que se incluyeron los pacientes mayores de I8 años atendidos entre enero de 2017 y diciembre de 2018 por presentar hernias inguinales, que requirieron tratamiento quirúrgico de urgencias, y que presentaban heridas sucias o contaminadas. Los criterios evaluados fueron: infección de la herida quirúrgica, morbilidad y mortalidad, necesidad de remoción de la malla y recurrencia de la hernia.

Resultados. Diez pacientes fueron sometidos a cirugía de urgencias, requiriendo resección intestinal por necrosis: nueve de ellos, por hernias estranguladas y, uno, por apendicitis perforada. En uno de los diez pacientes, se consideró sucia la herida por presentar necrosis intestinal y perforación. Los nueve restantes presentaban necrosis intestinal sin perforación, por lo cual se consideraron heridas contaminadas. La infección de la herida ocurrió en I/IO pacientes con infección del sitio operatorio superficial; la eliminación de la malla no fue necesaria en ningún paciente durante todo el período de estudio. No se observaron recidivas y no hubo mortalidad.

Conclusión. El uso de malla de polipropileno para la corrección de hernias inguinales, en pacientes con heridas sucias o contaminadas, es efectivo y seguro, con una morbilidad aceptable y buenos resultados a corto plazo.

Palabras clave: hernia inguinal; herniorrafia; mallas quirúrgicas; prótesis e implantes; intestino delgado; infección de la herida quirúrgica.

Fecha de recibido: 21/05/2019 - Fecha aceptación: 17/10/2019

Correspondencia: Mauricio Pedraza, Carrera 72 N 81-55, Bogotá, D.C., Colombia Teléfono (301) 3709557

Correo electrónico: mpedraza93@gmail.com

Citar como: Mendoza-Zuchini, Pedraza-Ciro M, Sánchez-Ussa S, Cabrera LF, Pulido J, Gómez D. Uso de mallas de polipropileno en el reparo de la hernia inguinal con herida sucia o contaminada. Rev Colomb Cir. 2020;35:51-6. https://doi.org/10.30944/20117582.585.

Este es un artículo de acceso abierto bajo una Licencia Creative Commons - BY-NC-ND https://creativecommons.org/licenses/by-ncnd/4.0/deed.es 


\begin{abstract}
Introduction: Polypropylene mesh hernioplasty is the technique of choice for the repair of inguinal hernias. Currently, there is controversy about this technique in patients with dirty or contaminated wounds. However, evidence in the medical literature has shown its use can be safe. The authors present their experience in the use of polypropylene meshes in contaminated surgery to close inguinal hernias.
\end{abstract}

Methods: A retrospective observational study was conducted in patients older than I8 year-old between January 2017 and December 2018. Were included those with inguinal hernias, requiring emergency surgical treatment, and presented contaminated or dirty wounds. The inclusion criteria were infection of the surgical wound, the need for removal of the mesh removal and recurrence of the hernia.

Results: Ten patients underwent emergency surgery, requiring bowel resection for necrosis: nine of them for strangulated hernias and, one for perforated appendicitis. In one of the ten patients, the wound was considered dirty due to intestinal necrosis and perforation. The remaining nine had intestinal necrosis without perforation, so they were considered contaminated wounds. Wound infection occurred in I/IO patients (IO\%) with superficial operative site infection; mesh removal was not necessary in any patient during the entire study period. No recurrence was observed and there was no mortality.

Conclusion: The use of polypropylene mesh for the correction of inguinal hernias in patients with contaminated and dirty wounds is effective and safe, with acceptable morbidity and good short-term results.

Keywords: hernia, inguinal; herniorrhaphy; surgical mesh; prostheses and implants; intestine, small; surgical wound.

\section{Introducción}

La herniorrafia inguinal es uno de los procedimientos realizados más frecuentemente a nivel mundial, con cerca de 20 millones de operaciones al año. La prevalencia de la hernia inguinal en la población general aumenta con la edad; es de un poco menos del I \% en el grupo de 45 a 64 años y, de más del I, $5 \%$, en el grupo de más de 75 años ${ }^{\text {. }}$

Este procedimiento ha ido evolucionando con el tiempo y la hernioplastia con malla se ha convertido en la técnica de elección para el reparo de las hernias inguinales, logrando mejores resultados a largo plazo, ya sea por vía anterior o posterior, abierta o por laparoscopia; la técnica de Liechtenstein es la más frecuentemente utilizada a nivel mundial. Se considera que se previene una recidiva herniaria por cada 46 pacientes operados con malla, en comparación con los operados sin malla ${ }^{2}$.

En las últimas guías internacionales para el manejo de hernias inguinales publicadas por The Hernia Surge Group, se recomienda que las hernias inguinales se reparen con malla, con un grado de recomendación fuerte ${ }^{3}$. Aunque el reparo de las hernias inguinales con prótesis (mallas) se ha convertido en el procedimiento de elección para este tipo de hernias, siempre ha existido controversia en cuanto a si se deben usar las prótesis en las heridas quirúrgicas clasificadas como sucias o contaminadas por perforación o estrangulamiento del contenido herniario, por el alto riesgo de colonización e infección del material protésico.

Durante los últimos años, han surgido nuevos estudios en los cuales se ha demostrado el bajo riesgo de infección del material protésico en pacientes con heridas sucias o contaminadas, por lo que se presenta una revisión de la literatura.

\section{Métodos}

Se realizó un estudio retrospectivo y observacional de los pacientes que fueron sometidos a hernioplastia inguinal con malla, con heridas sucias o contaminadas. A todos los pacientes se les realizó un reparo de la hernia inguinal por vía posterior preperitoneal con la técnica descrita por Nyhus ${ }^{4}$, para la corrección con malla de hernias inguinales y crurales (figura I). 
En todos los casos se practicó una incisión suprapúbica lateral, se ingresó al espacio preperitoneal y se disecaron los componentes de la hernia; en el caso de los pacientes masculinos, se disecaron cuidadosamente los elementos del cordón espermático y, en el caso de la paciente, se disecó el ligamento redondo, y se repararon. Se disecaron y preservaron los vasos epigástricos cuando la alteración anatómica causada por la hernia lo permitió. Se redujo la hernia y se abrió el saco, identificando su contenido.

En los pacientes con necrosis de la pared intestinal y en el paciente que requirió apendicectomía, la resección se realizó en su totalidad con sutura mecánica de forma localizada y por la misma herida suprapúbica. En los casos en que se requirió, se redujo la anastomosis intestinal dentro del abdomen con posterior ligadura o cierre alto del saco herniario. Se colocó una malla de polipropileno de IO x I5 $\mathrm{cm}$ y se fijó al ligamento de Cooper con sutura separada de polipropileno desde la espina del pubis hasta la vena femoral. Se formó un nuevo anillo para permitir el paso del cordón espermático o del ligamento redondo. Se cerró la aponeurosis y la piel con sutura absorbible. En ningún caso se usaron antibióticos o sustancias antisépticas en la prótesis.

En el caso de perforación con peritonitis fecal, en el momento de cierre de la pared abdo-

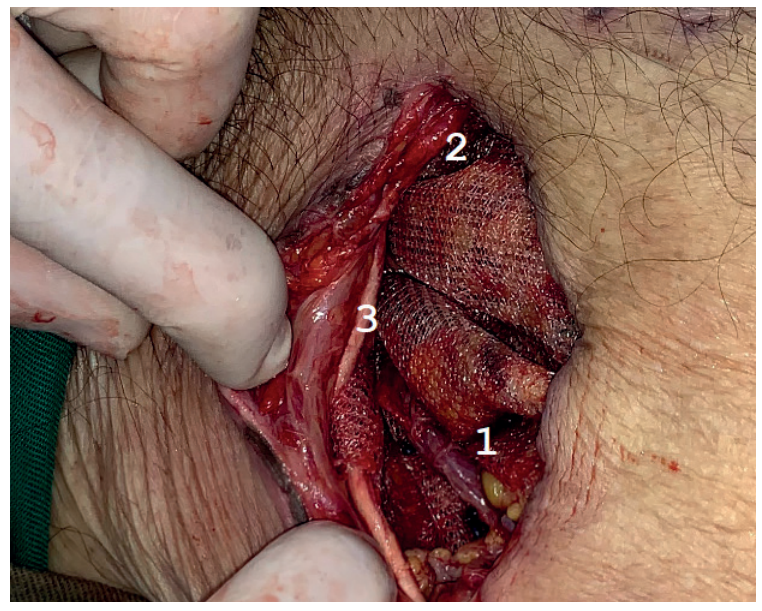

Figura 1. Malla en el espacio preperitoneal: 1. Elementos del cordón a través de la malla por el nuevo anillo. 2. Músculo recto abdominal. 3. Aponeurosis del músculo oblicuo mayor minal y la reparación de la hernia inguinal, se dejaron dos drenajes, uno en la cavidad abdominal y otro en la región inguino-crural (figura 2). El seguimiento mínimo en todos los pacientes fue de seis meses, sin detectarse recurrencias.

\section{Resultados}

Diez pacientes fueron sometidos a herniorrafia inguinal de urgencias, con uso de malla, ocho con necrosis intestinal y uno con apendicitis (figura 3) en quienes se consideró contaminada la herida

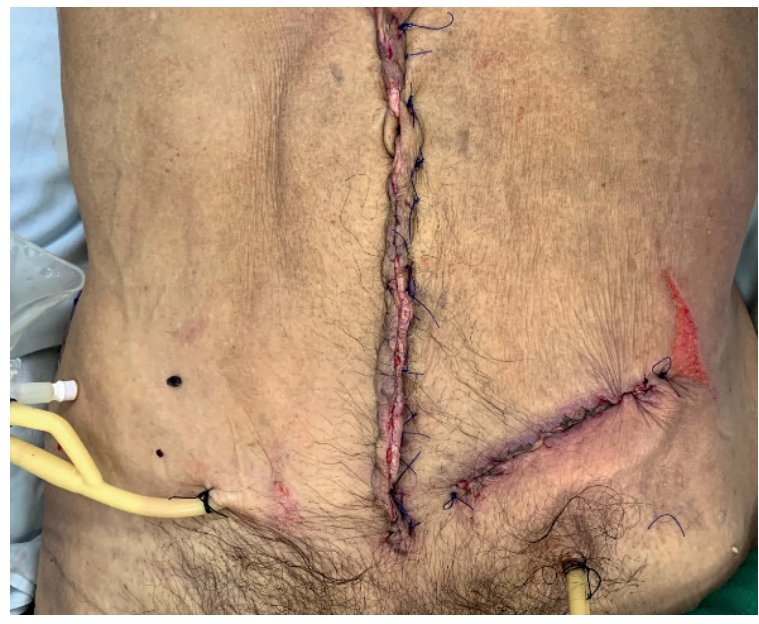

Figura 2. Drenaje posoperatorio después del cierre de la pared abdominal y herniorrafia inguinal con malla en una herida sucia por perforación asociada a peritonitis fecal

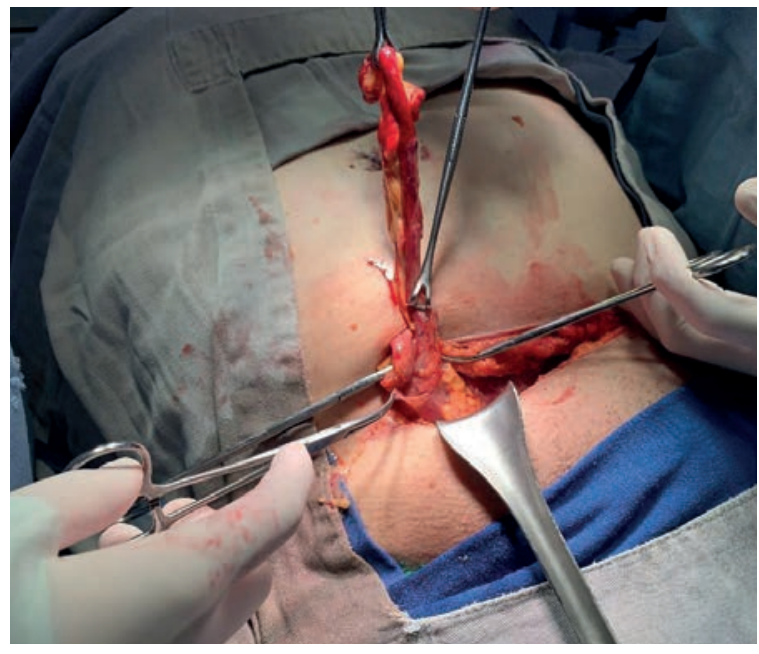

Figura 3. Hernia de Garengeot, apendicitis aguda fibrinopurulenta en hernia crural encarcelada 
$(\mathrm{n}=9)$, y uno con necrosis y perforación en quien se consideró sucia la herida. Ocho pacientes correspondieron al sexo masculino y 2 al femenino. La edad osciló entre los 56 y los $7 \mathrm{I}$ años, con una edad media de 63,5 años. El resumen de las características sociodemográficas y los resultados clínico-quirúrgicos se resumen en la tabla I.

Todos los abordajes se hicieron por vía preperitoneal lateral suprapúbica. En los 9 casos con herida contaminada (incluyendo el de apendicitis aguda) y sin evidencia de perforación intestinal, se practicó herniorrafia inguinal con malla en único tiempo quirúrgico. Cuatro pacientes requirieron resección y anastomosis intestinal. Un caso requirió laparotomía, además del abordaje preperitoneal, para lavar la cavidad abdominal por peritonitis fecal, y revisión quirúrgica a las 72 horas en la cual se llevaron a cabo la herniorrafia inguinal con malla y el cierre de la pared abdominal (figura 2).

La estancia hospitalaria fue de 2 a io días, con un promedio de 5 días. Solo el paciente de la herida sucia desarrolló infección superficial del sitio operatorio, que fue manejada médicamente con antibióticos. Ningún paciente requirió reintervenciones para retirar el material protésico y no se documentaron recidivas en un seguimiento de hasta I8 meses.

\section{Discusión}

A medida que se ha implementado el uso de las mallas en la reparación de las hernias inguinales, la infección de la prótesis ha sido tema de gran discusión. Siempre ha existido temor por parte de los cirujanos al colocar mallas en campos quirúrgicos sucios o contaminados, por el alto riesgo de infección. Se considera, además, que aproximadamente un tercio de las recidivas herniarias pueden ser secundarias a procesos infecciosos ${ }^{5}$. A pesar de esto, se considera indicado el uso de mallas en pacientes con heridas quirúrgicas clasificadas como contaminadas (riesgo de infección del 6,4\%) y en heridas sucias (riesgo de infección del 7,I \%), debido a que la malla reduce significativamente el riesgo de recidiva y a que la misma infección del tejido es una causa importante de recidiva.
Tabla 1. Características sociodemográficas de los pacientes operados y los resultados clínico-quirúrgicos

\begin{tabular}{|c|c|}
\hline Variable & Valor \\
\hline \multicolumn{2}{|l|}{ Sexo, n } \\
\hline Femenino & 2 \\
\hline Masculino & 8 \\
\hline \multicolumn{2}{|l|}{ Edad (años) } \\
\hline Promedio & 63,5 \\
\hline Rango & $56-71$ \\
\hline \multicolumn{2}{|l|}{ Abordaje, $n$} \\
\hline Preperitoneal & 9 \\
\hline Anterior & 0 \\
\hline Laparotomía & 1 \\
\hline \multicolumn{2}{|l|}{ Estancia hospitalaria (días) } \\
\hline Promedio & 5 \\
\hline Rango & $2-10$ \\
\hline \multicolumn{2}{|c|}{ Tiempo quirúrgico de la colocación de malla, n } \\
\hline Primer tiempo & 9 \\
\hline Segundo tiempo & 1 \\
\hline Necesidad de resección intestinal n (\%) & 4 \\
\hline \multicolumn{2}{|l|}{ Complicaciones, $\mathrm{n}(\%)$} \\
\hline Infección del sitio operatorio & 1 \\
\hline Necesidad de retirar la malla & 0 \\
\hline Necesidad de reintervención & 0 \\
\hline Recidiva & 0 \\
\hline
\end{tabular}

Actualmente, no hay una recomendación fuerte para el uso de prótesis en el reparo de hernias inguinales en heridas sucias o contaminadas. En el Current Problems of Surgery de 2008, se considera como contraindicación para el uso de mallas en reparo de hernias inguinales, la contaminación de la herida quirúrgica, pero también se informa que las mallas de polipropileno (usadas más en años anteriores, pero aún vigentes), parecen funcionar bien en presencia de infección, observándose una adecuada granulación de estas heridas, luego de drenada y tratada la colección y la infección, sin necesidad de retirar la malla ${ }^{6}$.

Posteriormente, se recomendó el uso de mallas biológicas con la teoría de que eran menos propensas a la infección, pero el seguimiento no fue favorable y se demostraron resultados negativos a largo plazo en los pacientes con heridas contaminadas ${ }^{7}$.

Las últimas guías publicadas para el manejo de las hernias inguinales, recomiendan el uso de mallas de polipropileno en las heridas limpias o 
contaminadas en cirugías de urgencias (grado de recomendación: débil), no recomiendan el uso de mallas en heridas sucias o contaminadas (grado de recomendación: débil), pero sí recomiendan el uso de mallas en casos de pérdida de tejidos o por alto riesgo de complicaciones, como las recidivas; es necesario evaluar el riesgo y el beneficio en este último tipo de pacientes ${ }^{3}$.

Infortunadamente, son muy pocos los estudios en los cuales se compara el uso de malla con su omisión, en pacientes con heridas contaminadas o sucias. La mayoría de los estudios se han realizado en pacientes con hernias ventrales e de incisión. En un metaanálisis de 20I4, se evaluó el uso de las mallas en el reparo de la hernia inguinal estrangulada; se incluyeron siete artículos, con un total de $4 \mathrm{I} 3$ pacientes en los que no se practicó resección intestinal. Se documentó un total de 26 casos de infección de sitio operatorio, 7 infecciones de sitio operatorio en el grupo de corrección quirúrgica con malla y los I9 restantes en el grupo en el que no se usó malla; la diferencia no fue estadísticamente significativa (odds ratio, $\mathrm{OR}=0,46, \mathrm{IC}_{95} \%$ O,20-I, O7).

También, se comparó la infección del sitio operatorio en 5 estudios con 22I pacientes, en los que se corrigió la hernia con malla y sin malla, pero en quienes, además, fue necesaria la resección intestinal; no se encontró una diferencia estadísticamente significativa entre los dos grupos.

En el mismo metaanálisis, en 3 estudios con 260 pacientes, se reportaron tasas de recurrencia significativamente más bajas en favor del grupo de reparación con mallas $\left(\mathrm{OR}=\mathbf{0}, 2 \mathrm{O} ; \mathrm{IC}_{95} \%\right.$ $0,05-0,78$ ) con 27 recurrencias: dos en el grupo con mallas y 25 en el grupo sin mallas ${ }^{8}$.

En un estudio realizado en 65 pacientes que fueron sometidos a reparación de hernia inguinal en un ambiente hostil (base aérea estadounidense en una provincia de Afganistán), que incluían 2 casos de hernias inguinales traumáticas en combate, se informó de 46 hernias corregidas con malla y i 9 sin malla, con o \% de infección del sitio operatorio asociado con el uso de malla, sin evidencia de recurrencia a un año de seguimiento y sin requerir ningún tipo de reintervención adicional. La incidencia total de complicaciones (dolor crónico) fue del $2 \%$ en el grupo con malla y de $5 \%$ en el grupo sin malla 9.

Otro estudio más reciente incluyó 30 pacientes ( 22 hombres y 8 mujeres) con hernias estranguladas, los cuales se sometieron a cirugía de urgencia con resección intestinal y anastomosis. Los 30 casos incluyeron 2I hernias inguinales, siete en la incisión, una femoral y una umbilical. En I2 de los 30 casos, las heridas se consideraron sucias por contenido intestinal debido a perforación, mientras que, en los 18 casos restantes, se clasificaron como contaminadas. La infección del sitio operatorio ocurrió en 8 (26,67 \%) pacientes, los cuales fueron tratados con antibióticos y medidas locales. La eliminación de la malla no fue necesaria en ningún paciente durante todo el período de seguimiento y se documentaron solo dos $(6,7 \%)$ recidivas; una hernia inguinal y una de la incisión; no hubo mortalidad. El tipo de malla que se utilizó fue la de polipropileno ${ }^{\text {Io }}$.

Estos resultados son similares a los observados en el presente estudio, en el cual, uno de cada cuatro pacientes desarrolló infección del sitio operatorio, pero que fue manejado médicamente con total éxito y sin necesidad de retirar el material protésico en ninguno.

En otro estudio con 257 pacientes que fueron sometidos a cirugía de urgencia por hernia inguinal complicada, 45 ( $17,5 \%)$ casos requirieron una resección intestinal y, en solo el I5 \% de estos casos, se colocó una malla sintética. El 3,6\% de la totalidad de los pacientes tuvo una infección en el sitio operatorio y el uso de la malla no se asoció con infección del sitio quirúrgico $(\mathrm{OR}=\mathrm{I}, 83$, $\mathrm{p}=\mathrm{o}, 49)$ o con complicaciones perioperatorias $(\mathrm{OR}=\mathrm{I}, 02 ; \mathrm{p}=\mathrm{O}, 96)$. Con una mediana de seguimiento de 43 meses, no hubo infecciones de la malla y las tasas de recurrencia fueron similares entre el uso de mallas y las reparaciones de tejidos: $6,3 \%$ frente a $6,8 \%(\mathrm{p}=0,9 \mathrm{I})^{\text {II }}$.

A pesar de que exista el riesgo de infección del material protésico en la reparación de las hernias inguinales con malla en pacientes con herida sucia o contaminada y de que no se recomiende el uso de mallas en heridas sucias o contaminadas por parte de las sociedades in- 
ternacionales de hernias ni en las últimas guías del 20I8, los resultados en distintos estudios recientes demuestran que la malla sintética en este tipo de heridas sí puede traer beneficios para el paciente, como la disminución en la tasa de recurrencia de las hernias inguinales. Además, su uso parece ser seguro debido a que no hay mayor riesgo en cuanto a infección del sitio operatorio o morbilidad perioperatoria en el paciente ${ }^{12}$.

Entre las limitaciones de este estudio, se menciona el tamaño de la muestra, la cual no es lo suficientemente grande para llegar a una conclusión positiva sobre el uso de las mallas en pacientes con heridas sucias o contaminadas. No hay una recomendación clara por parte de los expertos en cuanto al uso de mallas en la reparación quirúrgica de urgencias de la hernia inguinal en pacientes con heridas sucias o contaminadas, aunque consideramos que parece ser seguro, con tasas similares de infección del sitio operatorio en comparación con las técnicas de reparación basadas en los tejidos y con menores tasas de recurrencias.

\section{Conclusión}

El debate entre el dogma y los datos continúa, por lo que se recomienda llevar a cabo un estudio clínico para comparar la seguridad y la eficacia. No obstante, mientras tanto, consideramos que se está acumulando evidencia a favor de la malla de polipropileno en las hernias con heridas sucias o contaminadas. Por nuestros resultados, se confirma que las mallas de polipropileno con macroporo para reparaciones de hernias con heridas contaminadas o sucias, son efectivas, seguras, con baja morbilidad y buenos resultados a corto plazo.

\section{Cumplimiento de normas éticas}

Consentimiento informado: Este estudio fue aprobado por el Comité de Ética de la institución. De acuerdo con la Resolución 008430 de 1993 del Ministerio de Salud de Colombia, se consideró que la investigación no tenía riesgo para los pacientes por ser una revisión retrospectiva de historias clínicas, por lo tanto, no se diligenció el consentimiento informado.

\section{Conflicto de interés: ninguno}

Fuente de financiación: este trabajo fue autofinanciado.

\section{Referencias}

I. Irwin T, McCoubrey A. Adult groin hernias. Surgery (Oxford). 20I2;30:290-5. https://doi.org/IO.IoI6/j.mpsur. 2012.02.0I4

2. Lockhart K, Dunn D, Teo S, Ng JY, Dhillon M, Teo E, et al. Mesh versus non-mesh for inguinal and femoral hernia. Cochrane Database Syst Rev. 201813;9:CDoII517. https://doi.org/IO.IO02/I465I858.CDoII5I7.pub2.

3. Simons MP, Smietanski M, Bonjer HJ, Bittner R, Miserez M, Aufenacker TJ, et al. International guidelines for groin hernia management. Hernia. 2018;22:I-I65. https://doi.org/I0.IO07/sI0029-0I7-I668.

4. Nyhus LM. Iliopubic tract repair of inguinal and femoral hernia: The posterior (preperitoneal) approach. Surg Clin North Am. 1993;73:487-99. https://doi. org/Io.Ioi6/Soo39-6Io9(I6)46032-9.

5. Gopal SV, Warrier A. Recurrence after groin hernia repair-revisited. International J Surg. 2013;II:374e-7. https://doi.org/IO.IOI6/j.ijsu.2013.03.012

6. Matthews RD, Neumayer L. Inguinal hernia in the 2Ist century: An evidence-based review. Curr Probl Surg. 2008; 45:26I-3I2. https://doi.org/IO.IO67/j.cpsurg.2008. OI.002.

7. Rosen MJ, Krpata DM, Ermlich B, Blatnik JA. A 5-year clinical experience with single-staged repairs of infected and contaminated abdominal wall defects utilizing biologic mesh. Ann Surg. 2013;257:99I-6. https:// doi.org/I0.I097/SLA.oboi3e3I8284987I.

8. Hentati H, Dougaz W, Dziri C. Mesh repair versus nonmesh repair for strangulated inguinal hernia: Systematic review with meta-analysis. World J Surg. 20I4;38:2784-90. https://doi.org/IO.IOO7/s00268-0I4-2710-0.

9. Kuckelman JP, Barron MR, Blair K, Martin MJ. The routine use of prosthetic mesh in austere environments: Dogma vs data. Am J Surg. 20I6;2II:958-62. https://doi. org/IO.IOI6/j.amjsurg.2016.02.005.

Io. Pandey H, Thakur DS, Somashekar U, Kothari R, Agarwal P, Sharma D. Use of polypropylene mesh in contaminated and dirty strangulated hernias: Shortterm results. Hernia. 20I8;22:IO45-50. https://doi. org/IO.IOO7/sIO029-0I8-I8II-3.

II. Tastaldi L, Krpata DM, Prabhu AS, Petro CC, Ilie R, Haskins IN, et al. Emergent groin hernia repair: A single center IO-year experience. Surgery. 2019;165:398-405. https://doi.org/IO.IOI6/j.surg.20I8.08.ooI.

I2. Montejo JE, Pisonero JJ, Delgado AD. Uso de malla de polipropileno en la hernioplastia por técnica de Lichtenstein. Revista Cubana de Cirugía. 20II;50:I47-54. 